International Journal of Current Advanced Research

ISSN: O: 2319-6475, ISSN: P: 2319 - 6505, Impact Factor: SJIF: 5.995

Available Online at www.journalijcar.org

Volume 6; Issue 3; March 2017; Page No. 2549-2554

DOI: http://dx.doi.org/10.24327/ijcar.2017.2554.0047

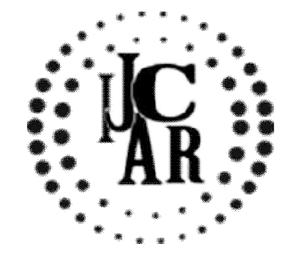

Research Article

\title{
COMPARISON BETWEEN 2D-PSIR AND 2D-IR MAGNETIC RESONANCE IMAGING IN THE EVALUATION OF MYOCARDIAL ENHANCEMENT
}

\author{
Ruchika $S^{*}$ and Prakashini K
}

Department of Radiodiagnosis and Imaging, Kasturba Medical College, Manipal

\section{A R T I C L E I N F O}

\section{Article History:}

Received $18^{\text {th }}$ December, 2016

Received in revised form $19^{\text {th }}$ January, 2017

Accepted $4^{\text {th }}$ February, 2017

Published online $28^{\text {th }}$ March, 2017

\begin{abstract}
A B S T R A C T
Aims and objective: Assessment of late gadolinium enhancement of myocardium in ischemic and non-ischemic cardiomyopathies holds a strong diagnostic and prognostic importance specially in terms of viability. The present study focused on comparison between the post contrast sequence for detecting infarcted or fibrosed myocardium tissue, for proper identification of LGE is important to narrow down the differential diagnosis. Our main aimwas to provide a comparison between 2D-PSIR and 2D-IR Magnetic resonance imaging in the evaluation of myocardial enhancement

Materials and Methods: 40suspected cases of ischemic \& non-ischemic cardiomyopathy underwent CMR with contrast.

For all cardiomyopathies their LGE pattern was assessed by 2DPSIR and 2D-IR sequences. Further image quality assessment was done at qualitative, inter-observer and quantitative levels for both the sequences

Results: Kendall Tau-b showed poor agreement between qualitative variability of overall image quality in PSIR \& IR, (Kendall's tau-b $=0.23^{* *}, \mathrm{p}=0.046$ ) PSIR showing significantly better over-all image quality which are independent of nulling time selection. There was significant difference in quantitative assessment that is CNR for PSIR v/s IR was $(p<0.001)$ A good inter-observer agreement for PSIR and IR image quality assessment (kendall tau-0.74, $\mathrm{p}<0.001$ ).

Conclusion: To our best knowledge, this is the first prospective studyto compare 2D-PSIR and 2D-IR sequences at $1.5 \mathrm{~T}$ with delayed contrast-enhanced cardiac magnetic resonance (MR) imaging value in the evaluation of ischemic and non-ischemic causes of cardiomyopathies, with respect to presence \&extent of LGE and viability assessment.PSIR revealed a better imaging quality with higher diagnostic confidence than IR images in terms of artifacts, sharpness and over all quality.
\end{abstract}

Copyright $₫ 2017$ Ruchika S and Prakashini K. This is an open access article distributed under the Creative Commons Attribution License, which permits unrestricted use, distribution, and reproduction in any medium, provided the original work is properly cited.

\section{INTRODUCTION}

Cardiac MRI (CMR) has become an important technique in the assessment of cardiomyopathies (Hundley, et al., 2010) (Wu, 2009) (DJ, et al., 2004) (WG, et al., 2007). Assessment of lategadolinium enhancement (LGE) has proved to be a diagnostic and an important prognostic tool in ischemic heart disease patients and has been trusted in making clinical decisions for suitability for coronary revascularization.

Cardiac MRI with LGEhas few limitations which are mainly the time consuming protocol and interferes of image quality due to motion artifacts. Consequently, optimizing the cardiac MRI acquisition protocols was important to the shorten acquition time still being able to achieve better quality image for diagnostic purposes.

\section{*Corresponding author: Ruchika S}

Department of Radiodiagnosis and Imaging, Kasturba Medical College, Manipal
Proper inversion time (TI) should be accurately selected for better contrast between the normal and abnormal areas in the myocardium.

With the target of stream lining our acquisition protocol to adapt increment in CMR indications, our study's fundamental aim was to compare the differences between 2D-PSIR (at 10 minutes) and late 2D-IR (at 15 minutes)- both qualitatively and quantitatively and to determine which one offers the better imaging and diagnostic qualities.

\section{MATERIAL AND METHODS}

This prospective study was conducted in the department of Radio diagnosis and Imaging, over period of 2 years. Institutional ethical committee's permission was obtained and all the patients were informed before taking their consent. Total number of patients in our study were 40. Inclusion criteria for our study was clinically suspected cases of nonischemic cardiomyopathies without coronary artery disease and Ischemic cardiomyopathies with coronary artery diseases 
confirmed with conventional angiography/ECG/lab reports. Exclusion criteriawas, acute myocardial infarction (requiring immediate management), deteriorated renal function, valve prosthesis \& pace maker or other conditions which were interfering with the patient's ability to comply withthe examination.

\section{Imaging protocol}

All patients underwent a $1.5 \mathrm{~T}$ cardiac MRI (Achieva1.5 T s, Phillips Medical Systems, The Netherlands) using 1 SENSE torso XL body coil with patient positioning being supine for all the cases. Routine conventional cine sequence were obtained followed by later gadolinium enhancement images from apex to the base of the heart the late gadolinium enhancement images were taken in short axis employing a T1weighted 2D inversion recovery fast spin echo. Post contrast phase sensitive inversion recovery sequence at 10 and 15 minutes for 2D-PSIR and 2D-IR respectively were required in short axis view. At approximately 12 mins after injection time we acquired look locker sequence allowing selection of a T1 to null normal myocardial signal (typically 200-300 msec), which is essentially required to achieve better image quality in case of 2D-IR sequence. Gadolinium based contrast agent at dose of $0.1 \mathrm{mmol} / \mathrm{kg}$ was used in all patients.

In additional to these, LGEsequences were acquired in the two cardiac planes that is (LV long-axis, 4-chamber)

Analysis or comparison of late PSIR and IR is done. Acquisition parameters are summarized in Table 1.

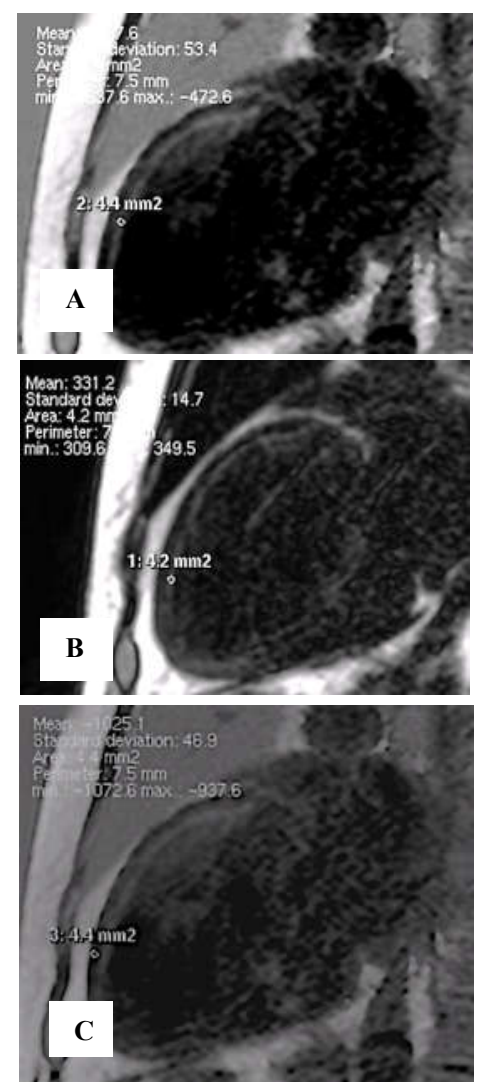

Fig. 1 Signal intensity measurement on subendocardial enhancement $(<25 \%$ thickness) involving apical- mid- basal anterior septal segment of left ventricle on VLA view of 2D-PSIR (a), 2D-IR (b) and healthy myocardium (c), with a $\sim 4.4 \mathrm{~mm} 2 \mathrm{ROI}$ in the wall (LGE).
Table no 1 Acquisition parameters for LGE sequences

\begin{tabular}{ccc}
\hline Sequence type & 2D-PSIR & 2D-IR \\
\hline TR & $6.1 \mathrm{~ms}$ & $6.1 \mathrm{~ms}$ \\
Flip angle & $25^{\circ}$ & $25^{\circ}$ \\
Field of view & $320 \mathrm{~mm}$ & $320 \mathrm{~mm}$ \\
Acquisition voxel size & $1.60 / 2.11 / 10.00 \mathrm{~mm}$ & $1.60 / 2.11 / 10.00 \mathrm{~mm}$ \\
Reconstruction matrix size & $1.33 \mathrm{~mm}$ & $1.33 \mathrm{~mm}$ \\
Slice number acquisition & 9 & 9 \\
Acquisition time per slice & $\sim 10 \mathrm{sec}$ & $\sim 10 \mathrm{sec}$ \\
Approximate acquisition time & $1.55 \mathrm{~m}$ & $1.55 \mathrm{~m}$ \\
\hline
\end{tabular}

\section{LGE evaluation}

For 2D-PSIR and 2D-IR sequences assessment was done by qualitative assessment and quantitative assessment by two radiologists (one experienced- observer-1and one traineeobserver-2)

\section{Qualitative analysis}

The myocardium-LGE contrast \& margin sharpness, artifacts, and overall image quality for PSIR and IR sequence were graded on a 4-point grading scale by two separate radiologists. Scoring method being, 1 for poor, 2 for fair, 3 for good and 4 for excellent quality images. Using Kendall Tau test the agreement between the two was evaluated (has to be changed). The criteria for 4 point grading system is given in table 2. [Schultz, et al., 2016]

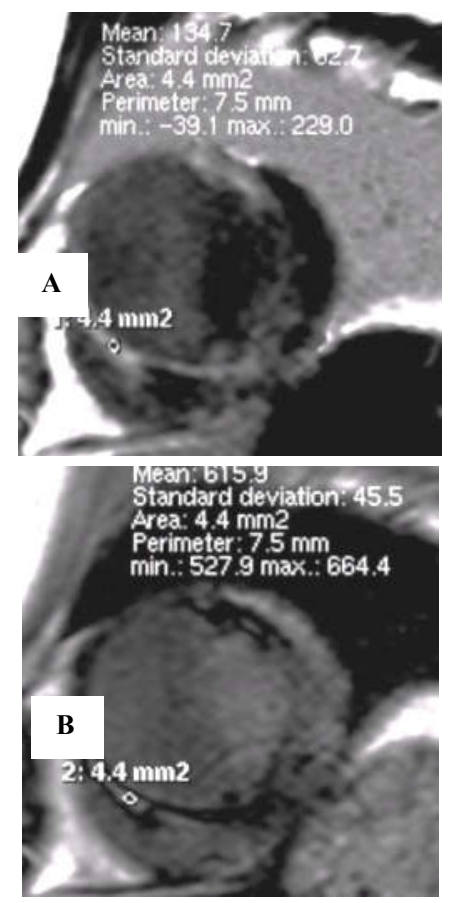

Fig. 2 In 59 year old male the signal intensity is measured on Short axis view of LV where LGE is seen in the mid antero-septal segment with $100 \%$ myocardial extent on 2D-PSIR (a), and 2D-IR (b), with a $\sim 4.4$ $\mathrm{mm} 2 \mathrm{ROI}$ in the wall (LGE). 
Table no 2 4-point Grading system

\begin{tabular}{|c|c|c|c|}
\hline Score & $\begin{array}{l}\text { LGE contrast and } \\
\text { margin sharpness }\end{array}$ & Artifacts & $\begin{array}{c}\text { Overall } \\
\text { image quality } \\
\end{array}$ \\
\hline 1 & Poor & $\begin{array}{l}\text { Severe, interfering with } \\
\text { the evaluation }\end{array}$ & $\begin{array}{l}\text { Poor, interfering with } \\
\text { the evaluation }\end{array}$ \\
\hline 2 & Fair & Moderate interfering & $\begin{array}{c}\text { Fair, moderate } \\
\text { interferences by the } \\
\text { artifacts }\end{array}$ \\
\hline 3 & Good & Mild interference & $\begin{array}{l}\text { Good, mild } \\
\text { interference }\end{array}$ \\
\hline 4 & Excellent & Minimal/ No artifacts & $\begin{array}{l}\text { Excellent, minimal to } \\
\text { no artifacts }\end{array}$ \\
\hline
\end{tabular}

Identification of LGE was grouped into two categories, Group 1 (score 3 \&4: good to excellent images) and into group 2 (score $1 \& 2$ : poor to fair images). In cases where score was given 2 by one observer and 3 by another, lower value was taken to avoid overestimation.

\section{Quantitative Assessment}

CNR value was calculated and recorded for both Late PSIR and IR sequence. Using ROI 4mm2 at LGE area (basal, mid and apical region) and in normal myocardium signal intensity and its standard deviation was measured.Using the following formula CNR was calculated. (Schultz, et al., 2016)

\section{CNR: LGE Signal - Myocardial Signal}

\section{(SD of LGE + SD of Myocardium) / 2}

For each manual ROI by drawing the LGE contours was done at the same level/place in a given case for both PSIR and IR sequences. Optimal adaptation of the window settings for each sequence was performed by the same operator so as to get an optimal and reproducible visualization of the LGE.

\section{Statistical analysis}

To measure the concordance between superior image quality between two LGE sequences and readers Cohen's Kappa was used [Cohen, 1960]. Hierarchical logistic regression models were used for comparisons between sequencesin order to take into account two random effects and thus intra-class variability for the reader and the subject effect.

\section{RESULTS}

Forty patients underwent a gadolinium enhanced cardiac MRI on our $1.5 \mathrm{~T}$ scanner over the period of 2 years.34patients exhibited LGE out of 40 patients. There were 15 patients of ischemic cardiomyopathy, with one being secondary dilated cardiomyopathy. Total non-ischemic cardiomyopathies were 21, out of which 12 cases were HCM, 4 were RCM, 1 DCM, 1 Myocarditis and 3 were cases of Takotsubo. The age group of the patients varied from 21-80 years with a mean of 57 years. Most of the patients were between $61-70$ years that is contributing to $37.5 \%$. Out of the total 40 patients, $27(67.5$ $\%)$ were males and $13(32.5 \%)$ were females.

Out of 34, 13 cases showed sub-endocardial late gadolinium enhancement, which contributed to $38.2 \%$ of the cases, 9 , showed transmural enhancement (26.4\%), 17- mid wall (50\%) and 2 epicardial $(5.8 \%)$.

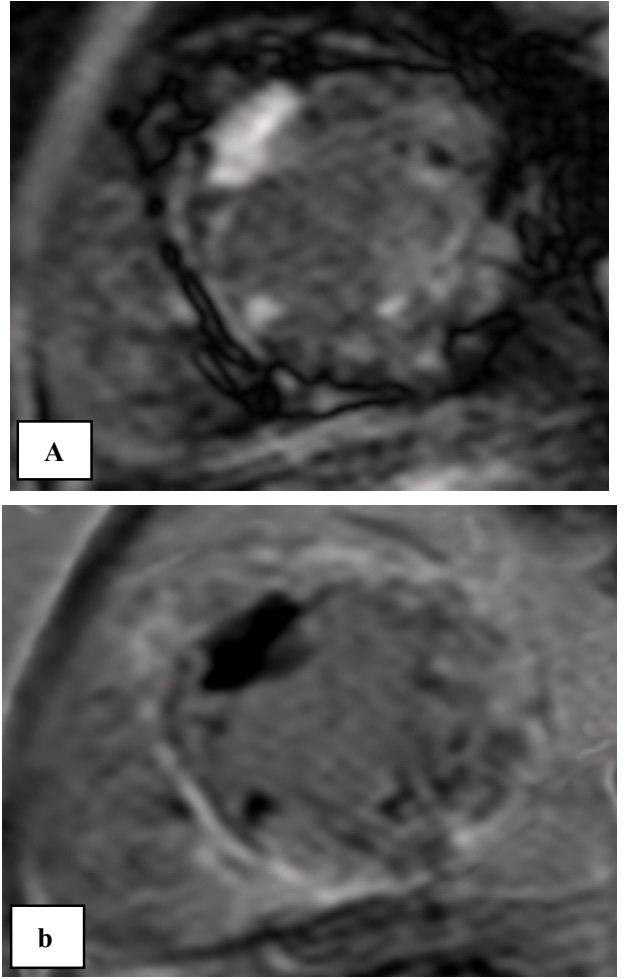

Fig. 3 Example of 29 year old male with restrictive cardiomyopathy secondary to amyloidosis diffuse patchy late gadolinium enhancement in the LV wall at mid cavity level with non-enhancing amyloid deposit involving the anterior segment on 2D-IR a) and on 2D-PSIR b).

\section{Comparison between 2d PSIR and 2d-IR sequence:}

\section{Qualitative evaluation}

Assessment of overall image quality on basis of sharpness \& contrast and artifacts showed that late 2D-PSIR sequenceswere significantly superior to 2D-IR sequences. Qualitative evaluation's result with a 4-level scale was summarized in Table 3 and 4. Inferential statisticalanalysis results are listed in Table 5. 2D-PSIR sequences are superior to 3D-IR. Qualitative variability for PSIR and IR images was; there was only significant disagreement between the two post contrast MRI sequences. 2D-PSIR sequence shows significantly higher number of images with better image quality (good to excellent) than images which were obtained by $2 \mathrm{D}-\mathrm{IR}$ sequence.

Table 3 Qualitative evaluation

\begin{tabular}{|c|c|c|c|}
\hline & & 2D-IR & \\
\hline 2D-PSIR & $\begin{array}{c}\text { Good to } \\
\text { excellent }\end{array}$ & $\begin{array}{c}\text { Poor to } \\
\text { fair }\end{array}$ & Total \\
\hline Good to excellent & 6 & 21 & 27 \\
\hline Poor to fair & 0 & 7 & 7 \\
\hline Total & 6 & 28 & 34 \\
\hline
\end{tabular}

Table 4 Descriptive analysis of qualitative evaluation

\begin{tabular}{ccc}
\hline & 2D-PSIR & 2D-IR \\
\hline Sharpness \& contrast & $3.03 \pm 0.63$ & $2.16 \pm 0.71$ \\
Artifacts & $2.08 \pm 0.6$ & $1.01 \pm 0.5$ \\
Overall image quality & $3.12 \pm 0.6$ & $2.16 \pm 0.63$
\end{tabular}



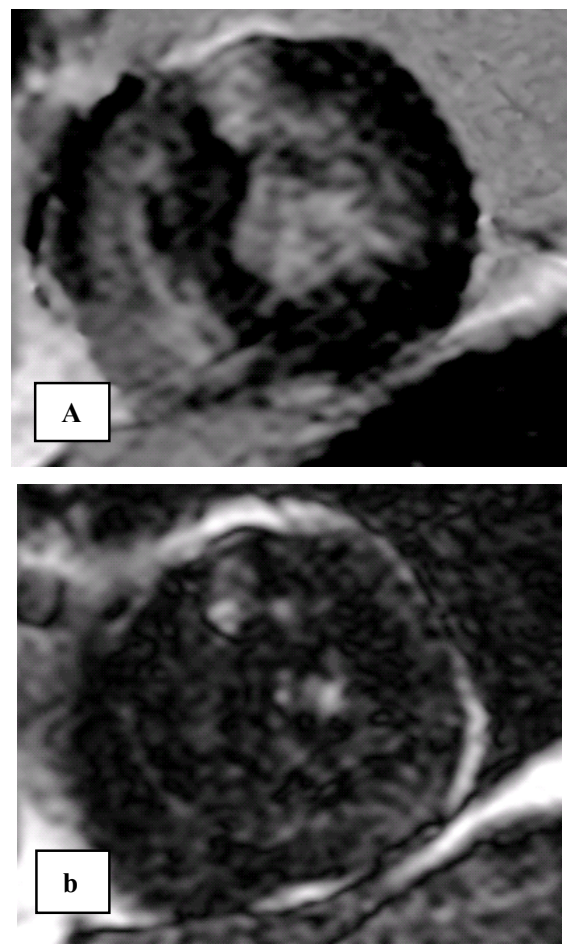

Fig. 4 Example of 57 year old male with hypertrophic cardiomyopathy showing patchy enhancement in the LV mid- wall involving antero septal segment ona) 2D-PSIR and b) 2D-IR

Table 5 Results of the inferential statistical analysis for the qualitative evaluation

\begin{tabular}{ccccc}
\hline & & $\begin{array}{c}\text { Sharpness \& } \\
\text { Contrast }\end{array}$ & Artifacts & Overall Image \\
\hline 2D- PSIR> & Mean of & 2.6278 & 2.2188 & 1.7813 \\
2D-IR & difference & Credible interval & $0.5739 ; 4.3643$ & $0.4508 ; 3.29370 .9763 ; 2.4142$ \\
& Likelihood & $1.000^{*}$ & $1.000^{*}$ & $1.000^{*}$ \\
\hline
\end{tabular}

Quantitative assessment confirmed the superiority of 2DPSIRover 2D-IR (Table $6 \&$ Table 7 Table 6 for Inferential analysis results for the quantitative evaluation).Mean Contrast-to-noise ratio (CNR) for all the 34 cases for 2DPSIR sequence was $20.77 \pm 12.12$ and for IR was $10.03 \pm 8.85$. There was a significant difference between their mean value with PSIR showing significantly higher values, almost more than twice the value obtained by IR sequences

Table 6 Quantitative evaluation

\begin{tabular}{ccc}
\hline & 2D-IR & 2D-PSIR \\
\hline CNR & $8.5 \pm 10.3$ & $18.9 \pm 13.2$ \\
\hline
\end{tabular}

Table 7 Results of the inferential statistical analysis for the quantitative evaluation

\begin{tabular}{ccc}
\hline & & CNR \\
\hline 2D- PSIR $>$ 2D- IR & Mean of difference & 0.7575 \\
& Credible interval & $0.0553 ; 0.1595$ \\
& Likelihood & $1.000^{*}$ \\
\hline
\end{tabular}

\section{Inter-observer agreement}

Kappa tests showed a strong agreement between the two readers on terms of artifacts and overall image quality, however there was a slight disagreement between the two readers concerning the sharpness and margins (0.6694 2DPSIR sequence and 0.4583 for the 2D-IRsequence). Disagreement was due toimages being scored 3 by one and 4 by the other one. Diagnostic quality image scored as 3/4 and poordiagnostic image quality scored as 1-2 were correctly assessed by both observers.

\section{DISCUSSION}

In our studycollation between different LGE sequences showedsuperiority at quantitative and qualitative levels for the late-PSIRover the late IR sequences.These results were in accordance with previous studies which were also carried out on 1.5 Tesla, the significance and importance of PSIR sequences for LGEimaging is well-established [(Chen, et al., 2013) (Kino, et al., 2011) (Kino, et al., 2009) (Robert, et al., 2013) (Elgeti, et al., 2007) (Huber, et al., 2006)]. Studies were carried outat 3T, by Kido, et al., 2014. who evaluated 56 patients, and showedno noteworthy difference in overall image quality between free-breathing 3D-PSIR and breathheld 3D-IR (R2=0.96) however they concluded 3D PSIR helped in detection of LGE in cases of non-ischemic cardiomyopathies whereas IR images over estimated LGE.

Morita, et al., 2013 did a study onthirty patients with hypertrophic cardiomyopathyat $3 \mathrm{~T}$ and found that myocardium-LGE contrast and overall image quality were significantly higher on 3D-PSIR thanon 2D-IR images $(\mathrm{p}<.001)$, with no consequential difference with respect to margin sharpness and artefacts.

This study helps us to come to a conclusion regarding which sequences provides better image quality without missing out on any segment showing late gadolinium enhancement. The superiority of PSIR over IR can be explained by the inherent characteristics of these two sequences [Kellman, et al., 2002]. In patients with ischemic \& non-ischemic cardiomyopathies, LGE cardiac MR is an important investigation to measure the irreversible myocardial injury or scar with a good prognosis in ischemic scar quantification and for planning further treatment. Further it helps in with dilated cardiomyopathy and hypertrophic cardiomyopathies in assessment of the LGE; its presence, extent and the future risk factors associated with the cardiomyopathy. Bright signal representing scar can be differentiated from the nullified myocardium by using $\mathrm{T} 1$ mapping or by another aided T1 scout (lock looker/ modified lock looker) sequence which provide an accurate inversion time at which the myocardium nullifies [Kim, et al., 2003]. For inversion recovery (IR) imaging to acquire a good quality image this technique of using accurate inversion time (TI) is a must hence a faster alternative called phase-sensitive inversion recovery (PSIR) has been developed which is not depended upon TI of myocardial nulling.

Inappropriate selection of the inversion time (TI) results in incomplete suppression of the myocardium. Most common error is the selecting a shorter TI, resulting in a subendocardial "ring of hypointensity" and a mid-myocardial zone of hyperintensity. These artifacts mimic true midmyocardial delayed enhancement that is seen in pathologies like sarcoidosis or DCM.In cases of restrictive cardiomyopathies where suppression of abnormal myocardium (amyloidosis is the prototypical example) causes hypointensity of abnormal myocardium. This will result in the poor quality of delayed enhancement images that is a hallmark of patients with amyloidosis hence important potential pitfalls of LGE result from incorrect inversion time selection. First, incorrect nulling of the myocardium reduces 


\section{Comparison between $2 d$-psir and $2 d$-ir magnetic resonance imaging in the evaluation \\ of myocardial enhancement}

the conspicuity of true myocardial delayed enhancement, potentially interfering with the diagnosisof underlying pathology, resulting in a false negative result and secondly, incomplete nulling due to a short inversion time, if not recognized as artifact, can be erroneously interpreted as diffuse mid-myocardial LGE, leading to a false positive result. The interpreting radiologist must be familiar with the appearance of deviations of the TI selection, and be aware of underlying conditions, particularly amyloidosis, that can cause difficulty in selecting the correct TI time. (David, et al., 2015)

Typical clinical scenario TI is selected to provide maximal contrast between normal and abnormal myocardium by completely nulling any signal from normal myocardium. TI selection determines the sensitivity for the detection of myocardial damage. It enables restoration of the voxel polarity since images are acquired in alternating heartbeats using a phase-sensitive reconstruction to rectify the tissue signal intensities caused secondary to inaccurate TI selection. The PSIR sequence counter balance any cutback in CNR due to TI changes and maintains signal as well as contrast consistent during scanning, avoiding the need for a narrow definition of optimal TI.

Accurate measurement and quantification is possible when there is a good contrast difference between normal and abnormal area with well defined margins and high signal intensity incomparision to normal remote myocardium

Thus late 2D-PSIR-breath hold sequence shows high spatial resolution, better image quality on terms of margin, artifacts and over-all image quality being acquired in a lesser duration of time without missing out any LGE areas.

In our study, late 2D PSIR at 10 minutes showed a high likelihood $(100 \%)$ of being superior to late $2 \mathrm{D}$-IR acquired at 15 minutes, in terms of LGE margin sharpness, CNR and overall image quality.

Bayesian inference analysis indicates superiorstatistic power and gives a probability evaluation of the qualitative difference between the sequences, a binary clustering of qualitative assessment data (score 1 and 2 grouped in category 2, score 3 and 4 grouped in category 1 with concordance analysis showing astrong agreement between the two radiologists. This result needs to be put intoperspective with the number of categories, which were two in our study, in case of increase in categories there is a lower concordance. Reason for slight disagreement in our study was predominately between scores of 3 and 4, which does not affect nor hamper the diagnostic value of the image quality.

\section{CONCLUSION}

Our study shows that on a 1.5T scannerlate 2DPSIRsequences were significantly superior to 2D-IR in terms of qualitative \&quantitative assessment of LGE. PSIR revealed a better imaging quality with higher diagnostic confidence than IR images in terms of artifacts, sharpness and overall quality.

PSIR has advantage over IR sequence in imaging diffuse infiltrative cardiomyopathies secondary to amyloidosis where the normal blood pull nulling before the myocardium is altered resulting in a false inversion time (TI)

It over comes one of the major drawbacks of IR sequence, its dependency on look locker sequence and saves times by avoiding need of repetitive acquisition of looksequence in order to acquire early (5mins) and late (10-15mins) LGE images.

Since PSIR is not dependable upon the timing (TI) of myocardial nulling it surpasses the limitation faced by IR sequences in terms of over all image quality which is hampered due to manual errors in proper selection of TI for IR sequence acquisition

\section{References}

1. Chen, X. et al., 2013. Three-Dimensional PhaseSensitive Inversion-Recovery Turbo FLASH Sequence for the Assessment of Left Ventricular Myocardial Scar in Swine. PLOS ONE 8, Volume 10, pp. 325-337.

2. Christensen, R., Johnson, W., Branscum, A. \&Hanson, T. E., 2010. Bayesian Ideas and Data Analysis: An Introduction for Scientists and Statisticians. s.l.:s.n.

3. Cohen, J., 1960. A Coefficient of Agreement for Nominal Scales. Educational and Psychological Measurement, Volume 20, pp. 37-46.

4. David, B. \& Stefan, L. Z., 2015. Inappropriate inversion time selection for late gadolinium enhancement imaging. Pearls and Pitfalls in Cardiovascular Imaging, pp. 146149.

5. DJ, P. et al., 2004. Clinical indications for cardiovascular magnetic resonance (CMR): Consensus Panel report. Eur Heart J, 25(21), pp. 1940-1965.

6. Elgeti, T. et al., 2007. Assessment of late gadolinium enhancement in nonischemic cardiomyopathy: comparison of a fast Phase-Sensitive Inversion Recovery Sequence (PSIR) and a conventional segmented 2D gradient echo recall (GRE) sequence--preliminary findings. Invest Radiol, 42(10), pp. 671-675.

7. Freedman, L., 1996. Bayesian statistical methods. BJM, 313(7057), pp. 569-570.

8. Huber, A. et al., 2006. Phase-sensitive inversion recovery (PSIR) single-shot TrueFISP for assessment of myocardial infarction at 3 tesla. Invest Radiol, 41(2), pp. 148-153.

9. Hundley, W. et al., 2010. ACCF/ACR/AHA/NASCI/SCMR 2010 expert consensus document on cardiovascular magnetic resonance: a report of the American College of Cardiology Foundation Task Force on Expert Consensus Documents. J Am Coll Cardiol, 55(23), pp. 2614-2662.

10. Kellman, P., Arai, A., McVeigh, E. \& Aletras, A., 2002. Phase-sensitive inversion recovery for detecting myocardial infarction using gadolinium-delayed hyperenhancement.. Magn Reson Med, 47(2), pp. 372-383.

11. Kido, T. et al., 2014. Three-dimensional phase-sensitive inversion recovery sequencing in the evaluation of left ventricular myocardial scars in ischemic and nonischemic cardiomyopathy: comparison to threedimensional inversion recovery sequencing. Eur $J$ Radiol, 83(12), pp. 2159-2166. 
12. Kim, R., Shah, D. \& Judd, R., 2003. How we perform delayed enhancement imaging. $J$ Cardiovasc Magn Reson, 5(3), pp. 505-514.

13. Kino, A. et al., 2011. Assessment of left ventricular myocardial scar in infiltrative and non-ischemic cardiac diseases by free breathing three dimensional phase sensitive inversion recovery (PSIR) Turbo FLASH. Int $J$ Cardiovasc Imaging, 27(4), pp. 527-537.

14. Kino, A. et al., 2009. Three-dimensional phase-sensitive inversion-recovery turbo FLASH sequence for the evaluation of left ventricular myocardial scar. AJR Am J Roentgenol, 193(5), pp. W381-W388.

15. Lunn, D. J., Thomas, A., Best, N. \& Spiegelhalter, D., 2000. WinBUGS - A Bayesian modelling framework.

16. Morita, K. et al., 2013. Comparison of 3D phasesensitive inversion-recovery and $2 \mathrm{D}$ inversion-recovery MRI at 3.0 $\mathrm{T}$ for the assessment of late gadolinium enhancement in patients with hypertrophic cardiomyopathy. Acad Radiol, 20(6), pp. 752-757.
17. Ntzoufras, I., 2009. Bayesian Modeling Using WinBUGS. s.1.:Wiley.

18. Robert, J. et al., 2013. Infarct quantification using 3D inversion recovery and $2 \mathrm{D}$ phase sensitive inversion recovery; validation in patients and ex vivo. $B M C$ Cardiovasc Disord, 13(110).

19. Schultz, A. et al., 2016. Late gadolinium enhancement cardiac imaging on a $3 \mathrm{~T}$ scanner with parallel RF transmission technique: prospective comparison of 3DPSIR and 3D-IR. European Radiology, 26(6), pp. 15471555.

20. WG, R. et al., 2007. Cardiovascular MRI: Its current and future use in clinical practice. Expert Rev Cardiovasc, 5(2), pp. 307-3021.

21. Wu, K. C., 2009. Variation on a theme: CMR as the "one-stop-shop" for risk stratification after infraction?. JACC Cardiovasc Imaging, 2(7), pp. 843-845

\section{How to cite this article:}

Ruchika S and Prakashini K (2017) ' Comparison between 2d-psir and 2d-ir magnetic resonance imaging in the evaluation of myocardial enhancement', International Journal of Current Advanced Research, 06(03), pp. 2549-2554.

DOI: http://dx.doi.org/10.24327/ijcar.2017.2554.0047 